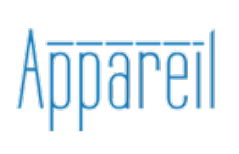

Appareil

$19 \mid 2017$

Friedrich A. Kittler : Esthétique et théorie des médias

\title{
La mort de l'auteur selon Friedrich Kittler
}

\section{Emmanuel Guez et Frédérique Vargoz}

\section{(2) OpenEdition}

\section{Journals}

Édition électronique

URL : http://journals.openedition.org/appareil/2561

DOI : 10.4000/appareil.2561

ISSN : 2101-0714

Éditeur

MSH Paris Nord

Référence électronique

Emmanuel Guez et Frédérique Vargoz, «La mort de l'auteur selon Friedrich Kittler », Appareil [En ligne], 19 | 2017, mis en ligne le 24 janvier 2018, consulté le 30 juillet 2020. URL : http://

journals.openedition.org/appareil/2561; DOI : https://doi.org/10.4000/appareil.2561

Ce document a été généré automatiquement le 30 juillet 2020.

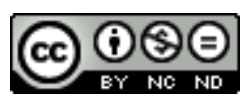

Appareil est mis à disposition selon les termes de la Licence Creative Commons Attribution - Pas d'Utilisation Commerciale - Pas de Modification 4.0 International. 


\title{
La mort de l'auteur selon Friedrich Kittler
}

\author{
Emmanuel Guez et Frédérique Vargoz
}

1 "Comme nous le savons tous, même si nous ne voulons pas nous l'avouer, aucun être humain n'écrit plus ${ }^{1}$ ». Les programmes informatiques en effet écrivent à notre place, ou tout au moins conditionnent notre écriture. Friedrich Kittler explique ainsi comment l'architecture des microprocesseurs est aujourd'hui réalisée par des logiciels fonctionnant eux-mêmes sur la base matérielle des architectures des ordinateurs antérieurs. L'écriture humaine est désormais une inscription électrique gravée dans le silicium de nos ordinateurs, c'est-à-dire un différentiel électrique.

2 La mort de l'auteur est d'abord la fin de l'écriture, tombée sous les coups de boutoir des média techniques, le gramophone, le film et la machine à écrire, soit l'ensemble des moyens d'enregistrement, de représentation et de traitement de l'information ayant ouvert la voie à une inscription ne passant pas par le stade de l'interprétation de l'information. Si nous n'écrivons plus, c'est parce que les média techniques, à partir du $\mathrm{XIX}^{\mathrm{e}}$ siècle, ont pu capter du réel des données qui échappent à la perception humaine : le gramophone enregistrait des oscillations non perceptibles à l'oreille humaine, la machine à écrire, augmentant la vitesse d'écriture, permettait d'automatiser le geste d'écriture et d'extorquer à ce qui était devenu une machine humaine ce que la lente écriture manuscrite ne pouvait lui soutirer, les circuits intégrés de l'ordinateur traitent les données plus rapidement que n'importe lequel ou laquelle des calculateurs et calculatrices humains employés jusqu'à la fin de la Seconde Guerre mondiale. Michel Foucault, en conclusion de son article « Qu'est-ce qu'un auteur ${ }^{2}$ ? » maintes fois cité par Friedrich Kittler, affirme que l'interrogation sur la figure de l'auteur présente l'intérêt d'ouvrir à une analyse du sujet comme "fonction variable et complexe du discours", analyse qui ne partirait plus du sujet et de sa liberté, compris comme des instances originaires, mais du discours et de son ordre. Friedrich Kittler, quant à lui, s'intéresse à la décomposition média-technique du discours. Ce sont les formes de cette décomposition que nous nous efforcerons ici de retracer. 


\section{Les systèmes d'inscription : de la nature au réel}

3 Ce qui intéresse notamment Friedrich Kittler est la manière dont les média techniques, développés à la fin du $\mathrm{XIX}^{\mathrm{e}}$ siècle et au $\mathrm{Xx}^{\mathrm{e}}$ siècle ont affecté, dans sa nature même, le médium qu'ils concurrençaient: l'écriture imprimée. Ce passage est raconté avec beaucoup d'humour par Salomo Friedländer dans une nouvelle parue en 1916 et reproduite dans Gramophone, Film, Typewriter ${ }^{3}$. "Goethe parle dans le gramophone " imagine le télescopage des deux systèmes d'inscription ${ }^{4}$ : celui de 1800 , où le "UrAutor » du classicisme allemand laisse parler son âme, pour la transformer en «écrits gutenbergiens » et celui de 1900, où la voix enregistrée ne vient plus de l'âme mais des vibrations sonores, captées par un dispositif complexe (complètement fictif malgré la précision média-technique avec laquelle il est décrit par Friedländer ) et s'inscrivant sur le cylindre en rotation d'un gramophone. Dans la nouvelle de Friedländer, Anna Pomke, lectrice relevant encore du régime de lecture du début du XIX ${ }^{e}$ siècle, rêve de ressusciter la voix de Goethe. Cette voix, dit Kittler, la lectrice de 1800 l'entend entre les lignes, alors que sa lecture de type hallucinatoire la plonge dans un monde intérieur d'images et de sons qui se substituent au texte. Cette voix est celle du poète qui, à travers la lectrice, se découvre lui-même en même temps qu'il se révèle dans son individualité universelle et dont le " chant », comme il est écrit dans « La Dédicace » de Faust citée par Kittler, "pareil à une vieille fable à demi-perdue», évoquait «les vieilles amitiés, les premières amours » et retentissait aussi "pour des inconnus ». «Lors de la relecture des libertés poétiques inconscientes, le bonheur narcissique produit la "fonction auteur". La figure de l'auteur dans le système d'inscription 1800 n'est pas une fonction simultanée de l'écriture, mais un effet ultérieur de la relecture ${ }^{5}$ ».

Brûlant de désir pour Anna et cherchant à la satisfaire, l'ingénieur Abnossah Pschorr ne peut néanmoins proposer qu'une solution d'homme des média techniques de 1900. Après avoir fabriqué un faux larynx à partir du moulage du squelette de Goethe, il le place dans la chambre de la maison du Frauenplan à Weimar en le connectant à un microphone, à un gramophone et à un soufflet, afin d'aspirer les ondes que le grand homme a produites lors de ses conversations et qui «quoique naturellement extrêmement atténuées doivent encore vibrer...6 ». Ce qui s'élève au contraire est un discours dépourvu de sens, produit par l'échantillonnage aléatoire des vibrations encore présentes dans la pièce. L'illustre auteur n'est plus rien d'autre que le réel luimême, en tant qu'il se grave, indépendamment de toute intention et en deçà de toute perception, sur le cylindre du gramophone. Ce faisant, Abnossah Pschorr célèbre l'exact contraire de la littérature classique et romantique.

Seuls les média techniques en effet, dont les informations empruntent des canaux physiques, fonctionnent avec un bruit de fond intrinsèque et sont à même de capter ce bruit blanc qu'aucune écriture ne peut enregistrer, le flou dans l'image cinématographique ou le bruit de l'aiguille du gramophone, qui détermine le rapport signal sur bruit. [...] Tel est le prix à payer pour que leurs reproductions soient aussi des émanations de ce qui est représenté, ce bruit de fond étant produit par les canaux mêmes empruntés par chacun des média ${ }^{7}$.

5 La voix de Goethe, qui pour Anna Pomke était la promesse d'une coïncidence entre le réel, la parole et l'écriture n'est plus que le bruit blanc des média. Friedrich Kittler s'appuie ici sur les analyses de Jacques Derrida, qui montrent dans la Grammatologie $e^{8}$, comment le mot a été compris comme l'unité élémentaire et indécomposable du 
signifié et de la voix, du concept et d'une substance d'expression transparente. La voix s'entend en une auto-affection pure, au plus proche de soi, comme l'effacement absolu du signifiant, elle n'est pas considérée comme un médium, comme une médiation, mais comme ce qui coïncide avec la nature. La voix signifiante de la nature se dit au cœur du poète, qui l'écrit, sans qu'il y ait solution de continuité entre la nature et l'écriture. L'auteur en 1800 n'est donc pas un sujet, au sens de substrat autonome et autocréateur. La critique kantienne est passée par là : le sujet transcendantal n'est rien d'autre qu'une place vide, un fondement a priori. Il n'existe que dans la représentation qu'il unifie. Mais il s'ancre dans une parole première, dans une unité première entre la parole et la nature, dont il n'est que le déploiement.

Le médium de la poésie est précisément ce par l'intermédiaire de quoi l'esprit humain parvient à la conscience et soumet ses représentations à une liaison arbitraire et à leur expression, le discours. Elle n'est en conséquence pas liée aux objets, mais elle crée les siens propres. Elle est le plus complet de tous les arts, et en même temps l'esprit universel présent en chacun d'eux'.

6 Cette unité, cette parole première, Kittler en retrace la genèse à partir d'une analyse de la réforme de l'alphabétisation en Allemagne au tournant du $\mathrm{XIX}^{\mathrm{e}}$ siècle. La réforme pédagogique instituée par l'introduction de la méthode phonétique place la voix - et plus particulièrement la voix de la mère - au centre de l'apprentissage simultané de la lecture et de l'écriture. Il ne s'agit plus pour un pédagogue comme Heinrich Stephani, par exemple, d'enseigner les lettres de l'alphabet, mais des sons élémentaires et purs. Ceux-ci ne sont pas aléatoirement construits à partir des lettres (comme cela deviendra le cas un siècle plus tard avec l'introduction de l'ânonnement des ba-be-bi et autres $p a-$ pe-pi), mais sont des signifiés minimaux. Il ne s'agit pas d'apprendre des syllabes dépourvues de sens, mais des sons immédiatement signifiants.

Pour la première fois avec Rask, Grimm et Bopp, le langage (bien qu'on ne cherche plus à le ramener à des cris originaires) est considéré comme un ensemble d'éléments phonétiques [...] Toute une mystique est en train de naître : celle du verbe, du pur éclat poétique qui passe sans trace, ne laissant derrière soi qu'une vibration ${ }^{10}$.

7 C'est une révolution du point de vue du privilège de la vue et des lettres de l'alphabet, " car les lettres, avec leur nom, perdent leur statut ${ }^{11}$ ». C'est aussi l'assomption d'une certaine figure de l'auteur : c'est dans l'écriture de cette voix primordiale de la mèrenature que le scripteur devient écrivain et accède à lui-même.

Mais pour Goethe l'expérimentation se termine mal : Anna Pomke, sous le ravissement hallucinatoire du " puissant organe » du poète de Weimar, en oublie Abnossah qui s'en offusque et jette son dispositif sous les roues d'un train. Fin de l'histoire ou plus précisément fin du système d'inscription 1800 : la lectrice se marie à un ingénieur et cesse d'halluciner le monde de la poésie entre les lignes : l'ingénieur tue l'auteur et la voix de la nature se tait. On comprend alors pourquoi, dans l'expérimentation, le discours de Goethe avait commencé avec des sifflements, des raclements, des grincements, et s'était achevé avec des ronflements: la voix de Goethe ressuscitée n'était en réalité que le bruit du canal d'enregistrement et de transmission, né de la rencontre entre le réel et la matérialité du médium. L'illusion d'une voix qui dirait la nature sans médiation est détruite. «Les sillons des disques creusent la tombe de l'auteur ${ }^{12} »$.

9 En réalité, les média techniques ne tuent pas l'auteur, ils en font émerger une autre occurrence. C'est pour cette raison que Friedrich Kittler est fasciné par la psychanalyse 
qu'il considère comme une construction du sujet émergeant directement de la possibilité nouvelle d'enregistrer les voix et de filmer les corps. Le sujet freudien naît du gramophone. Freud en a quelques intuitions quand il compare la psychè à un appareil, l'inconscient du psychanalyste au récepteur téléphonique - ce qui n'échappe pas à Kittler ${ }^{13}$-, ou vante la « phonographo-fidélité » de ses oreilles. Le gramophone en effet enregistre tous les sons, sans exception et sans distinction entre les sons dotés de signification et les autres. Il inciterait même à parler sans censure de la conscience, selon l'expérience du psychiatre viennois Stransky qui, faisant parler (si possible vite et beaucoup) des sujets d'expérimentation dans le tube d'un phonographe, pour moitié des collègues, pour l'autre des patients, constate que les mêmes comportements sont observés chez les deux: les phrases ne se soucient plus de signifier, le langage fonctionne en toute autonomie. Et tandis que la secrétaire de Jacques Lacan, Gloria Gonzalez-Yerodia, appuie silencieusement sur le bouton du radiocassette en même temps que débute le séminaire (en 1975), Kittler lui-même se souvient ${ }^{14}$ que Lacan dit alors : « Nous sommes plus soumis à nos gadgets, microphones et média(s) que nous le pensons ». Incarnant, comme le sujet freudien, le système 1900, le patient lacanien est cependant plus directement le produit d'un dispositif cinématographique. Le cinéma implémente l'imaginaire où se construit l'unité du moi : c'est à partir de conditions média-techniques cinématographiques (et au moins par la médiation d'un miroir concave et d'un miroir plan, comme dans l'expérience du vase inversé reprise par Lacan à Brouass $\mathrm{e}^{15}$ ) que Lacan pense la manière dont le moi se forme. Les éléments imaginaires nés de l'image du miroir viennent s'assembler au moi réel et le processus d'unification du moi dans la psychanalyse est un processus d'ajustement du regard du patient à l'image du psychanalyste, elle-même réduite «à un miroir pur d'une surface sans accidents ${ }^{16}$ ", à une surface de projection / introjection cinématographique.

Ramenant Freud et Lacan à leurs conditions média-techniques, Friedrich Kittler souligne que les média sont les modèles privilégiés d'après lesquels les êtres humains conçoivent leurs appareils psychiques $^{17}$. On ne peut rien dire de l'âme ou de l'inconscient, sinon ce que nous en disent les média (du crayon jusqu'à l'ordinateur) à travers lesquels nous en prenons la mesure. Il en est de même de l'auteur. Il ne s'agit pas de se prononcer sur ce qu'il est ou n'est pas, mais de prendre la mesure de sa fonction à partir des média à travers lesquels il écrit, ou n'écrit plus.

\section{« Nos outils d'écriture participent à l'élaboration de nos pensées ${ }^{18}$ »}

11 À partir du tournant du XIX siècle, l'écriture n'est plus que l'impact d'un levier sur une feuille de papier. L'âme du poète classique et romantique n'était rien sans la plume et l'écriture manuscrite, prolongement de lui-même et de son corps, en lequel résonnait la voix qui le menait à l'écriture. Dans la nouvelle de E.T.A Hoffmann, Le Pot d'or, longuement commentée par Kittler dans Aufschreibesysteme 1800-1900, c'est par l'acte de copie d'un texte en sanscrit, hallucinant, à travers les étranges signes qui le composent, la femme qui l'appelle et qu'il désire, que l'étudiant Anselmus devient poète (échappant d'ailleurs ainsi à une carrière toute tracée de fonctionnaire). La parole de la nature doit, pour être audible, se traduire en écriture ${ }^{19}$. Quand bien même cette écriture n'inscrit le corps que comme symbole, quand bien même elle est appelée à s'effacer derrière le pur signifié qui se révèle à l'imaginaire du lecteur, c'est bien dans le flux continu des lettres 
écrites que l'auteur se manifeste, prend connaissance de lui-même et se révèle aux lecteurs comme poète. En 1800, l'écriture était une trace de l'âme qui s'inscrivait de façon continue sur le papier. Ce n'est plus le cas en 1900 : à la différence du poète de 1800 , qui ne transformait ses manuscrits en écrits gutenbergiens et ne faisait de ses écrits privés un médium universel d'accès à la poésie qu'en un second temps, l'auteur n'est désormais rien sans sa secrétaire ou sa machine à écrire.

Le plus souvent, il s'agit d'un ménage à trois : l'auteur qui n'est plus écrivain, la femmesecrétaire-écrivaine qui n'est pas auteur, et la machine à écrire, ce médium technique, par qui advient cette merveilleuse composition masculin-féminin. La littérature se transforme au fur et à mesure que le porte-plume est concurrencé par d'autres média. Avec l'apparition de la machine à écrire (et plus tard de l'ordinateur et des logiciels de traitement de texte), le geste d'écriture se découple du corps, l'écriture devient automatique. La machine à écrire consiste en un stock de signes différentiels, éléments discrets et combinables que celui qui tape sélectionne à l'aveugle en raison du décalage entre la vitesse de frappe et l'intention consciente ${ }^{20}$. De ce point de vue, l'écriture automatique des surréalistes n'est rien d'autre que la traduction de conditions médiatechniques : celui qui écrit à la machine ne sait pas ce qu'il écrit. Mais à la différence de l'auteur répétant le murmure de la nature, il n'est plus le porte-parole d'un signifié universel, il ne produit plus que des signifiants. C'est déjà ce que disait Roland Barthes qui, s'interrogeant sur la «mort de l'auteur", voyait dans Mallarmé celui par qui le langage parle ${ }^{21}$. Supprimer l'auteur au profit de l'écriture, c'est d'abord pour Barthes concevoir l'écriture, à la suite des linguistes, comme un processus d'énonciation " vide ${ }^{22}$ ", produit par un système différentiel de signifiants aussi bien que de signifiés se déterminant réciproquement. Friedrich Kittler, lui, voit dans l'auteur du Coup de dés celui qui fait ressortir le fait que le langage est encodé - «la littérature ne dit rien, sinon qu'elle se compose des vingt-six lettres de l'alphabet ${ }^{23} »$. L'époque des média techniques marque l'effondrement du système de signification : les signifiés minimaux sont remplacés par des syllabes dépourvues de sens, produites à partir d'unités discrètes, et l'auteur n'est plus une âme mais un joueur de dés dont on étudie le fonctionnement psycho-physique. La poésie classique laisse place à la poésie du jeune Christian Morgenstern, "matériau sans auteur ${ }^{24}$ ", produit de l'agencement sériel aléatoire de signifiants. Pris dans la tempête venue de l'effondrement du livre-roi, le navigateur-poète n'évitera le naufrage qu'en explorant les Nombres ${ }^{25}$. La mort de l'auteur est donc en définitive celle de l'écriture du pur signifié romantique, comme trace d'une pensée ou d'une sensibilité individuée. Avec les média techniques, l'écriture devient code. Encodage du bruit par le gramophone, encodage de la lettre par le télégraphe, encodage de la page par la machine à écrire.

Les média techniques attaquent en effet la poésie classico-romantique sur deux fronts. En même temps que la machine à écrire concurrence et discrétise l'écriture manuscrite, l'apparition du gramophone et du cinéma a pour conséquence que les corps et les sons ne sont plus contraints de passer à travers le filtre du symbolique pour être enregistrés et transmis, l'écriture et la lecture silencieuse n'ont plus le monopole de l'imaginaire. Le monde de la poésie, halluciné à travers les lignes du livre, ne peut pas concurrencer l'imaginaire des films ou les sons des chansons populaires. S'il y a, dans le système 1900, une mort de l'auteur, c'est d'abord celle d'une littérature qui avait le monopole de l'imaginaire. Le cinéma s'étant emparé de l'imaginaire, la littérature se scinderait alors en deux, entre la ernsthafte Literatur (littéralement littérature sérieuse) et la littérature 
populaire (littéralement, littérature de divertissement, Unterhaltungsliteratur). Pour Kittler, tous les ouvrages littéraires qui relèvent désormais de l'imaginaire appartiennent à la seconde catégorie. Les plus « grands » romanciers, comme Thomas Mann, n'échappent pas au déclassement, tant ces auteurs ne produisent finalement que la matière première de l'industrie du cinéma - ou de la radio. Mais que la littérature ait perdu sa primauté culturelle dans le système d'inscription 1900 ne signifie pas pour autant sa mort en tant qu'art. Face aux auteurs d'ouvrages immédiatement adaptables et adaptés au cinéma, les auteurs de la «E-Literatur », soucieux d'explorer le matériau propre de la littérature, considèrent le signe dans sa matérialité, jouant sur la typographie, la mise en page et les sonorités. L'art littéraire devient l'exploration des matérialités de l'écrit, de la lettre à la machine d'écriture - c'est-à-dire d'encodage elle-même. Les lettres deviennent une question technique ${ }^{26}$. Bruit, lettre, page, autrement dit la poésie, qui a perdu sa fonction élocutoire, devient un art sonore, graphique et visuel.

Lorsque Stefan George ${ }^{27}$ élabore sa propre typographie, la St-Georg Schrift, à partir de son écriture manuscrite, qu'il simplifie et généralise, supprimant les empattements et les contrastes entre pleins et déliés mais aussi adaptant les écarts entre les mots et les lettres à la longueur des vers, il prend acte du fait qu'un livre est d'abord du papier et des polices d'écriture, en même temps qu'il prend congé du lien intime qui unissait l'intériorité du poète romantique à son écriture manuscrite. La lettre écrite ne doit plus servir de tremplin pour l'imaginaire mais d'obstacle à la compréhension, accrochant le regard. «L'écriture ", écrit Kittler, « pénètre toujours plus profondément dans la zone graphique de son imagéité nouvelle et excentrique » : du Coup de dés de Mallarmé et des Calligrammes d'Apollinaire, qui, comme poèmes typographiques, ne sont que des tentatives d'élever l'écrivain à la hauteur du film et de la phonographie, jusqu'à la poésie concrète, cette poésie pure de machine à écrire ${ }^{28}$ ». La fragilisation de la toutepuissance de l'auteur dès les années $1920^{29}$ puis sa mise à mort dans les années 1960 par le structuralisme, n'est donc que le symptôme de la mort de l'auteur du système 1800 .

\section{Un auteur ausculteur}

Mais cette mort nous invite rétrospectivement à nous retourner sur nos systèmes d'inscription, pour penser l'auteur différemment, comme celui qui écouterait le médium parler en lui. Le Coup de dés lance le jeu de l'écriture littéraire au $\mathrm{xx}^{\mathrm{e}}$ siècle. L'auteur, sous peine de naïveté littéraire - autrement dit (sous peine) de ne servir que de corps à la machine, sans aucune conscience de ses déterminations - est désormais contraint de s'emparer des matérialités machiniques de l'écrit, graphiques, visuelles, sonores et tactiles. Aujourd'hui, avec les ordinateurs, il ne peut faire l'économie de comprendre - et jouer - avec la condition de toute écriture - le code et les nombres. L'auteur du système post-1900 n'est plus un scripteur (manuscrit comme dans le système 1800), ni un écrivain ou une écrivaine devenu(e) secrétaire des machines, mais l'ausculteur de son médium, l'explorateur du système d'inscription dans lequel il évolue. Au xxI siècle, il devient une sorte de médium du médium, cherchant à atteindre le cœur, voire le double ou quadruple cœur du processeur. Ainsi les poèmes de Mallarmé et de Morgenstern agissent-ils encore comme un programme pour la littérature du siècle passé et de ce début de siècle, ayant généré, en vrac, le spatialisme et la poésie concrète, le lettrisme et Isidore Isou, Henri Chopin et la poésie sonore, 
l'Oulipo et Raymond Queneau, Dick Higgins et Fluxus, ainsi que la littérature... numérique.

Mais avant d'en arriver là, « un point zéro de la littérature en 1900 est nécessaire ${ }^{30}$ » et ce " point zéro de la littérature définit un son inarticulée ${ }^{31}$ ». La littérature du $\mathrm{xx}^{\mathrm{e}}$ siècle commence non seulement avec le Nombre et l'éclatement des matérialités du livre mais aussi avec un son inarticulé, un signifiant sans signifié. Le Grand Lalula de 1905 par Morgenstern, préfigurant l'UrSonate de Kurt Schwitters, donne le la. Ce n'est pas un hasard si - expression consacrée de Kittler - cinq ans auparavant, le professeur de psychologie Hermann Ebbinghaus avait lu avec une voix forte au rythme d'une horloge "des rangées de syllabes sans signification ${ }^{32}$ " jusqu'à ce qu'il pût les réciter de mémoire, montrant ainsi que les compétences de mémoire sont mesurables. La mémoire des textes, dont le fonctionnement échappe à l'herméneutique, est constituée de combinaisons et de permutations ${ }^{33}$. Ebbinghaus avait-il de son côté connaissance du poème de Paul Scheerbart Kikakokú! Ekoraláps! publié en 1897 ? Est-ce une coïncidence ou la preuve que, avant l'OULIPO puis l'ALAMO ${ }^{34}$, et bien avant que Katherine Hayles ait pensé le posthumanisme comme une co-construction littéraire et techno-scientifique ${ }^{35}$, un nouveau système d'inscription affecte de concert aussi bien la science que la littérature et l'art? Le Grand Lalula, cette combinaison de matériels signifiants sans auteur (Kroklowafzi ? Semememi!), ne dit rien, sinon que la langue est du début à la fin une série de blablabla. D'emblée, la littérature du système 1900 est un «simulacre de folie ${ }^{36}$ », auquel les méthodes psychanalytique et structuraliste offriront les outils d'analyse nécessaires dans les années 1960-1970. "Pour le recyclage des déchets psychophysiques sans signification il n'est nul besoin d'auteur ${ }^{37}$ ", écrit Kittler. Tandis que dans le système 1800 le ma me mi mo mu se structurait en poème humaniste, le réseau de techniques et d'institutions qui lui succède génère - à la manière des générateurs de textes littéraires $\mathrm{du} \mathrm{xx}^{\mathrm{e}}$ siècle ${ }^{38}$ ou des bots littéraires et artistiques du $\mathrm{xxI}^{\mathrm{e}}$ siècle $^{39}-\mathrm{du}$ blablabla obscur et codé produit par les média techniques. Si l'auteur 1800 est devenu anecdotique, le corps du lecteur-récepteur est ce par quoi le sens peut émerger. La lecture attentive n'est plus silencieuse mais à crier, chanter et danser ${ }^{40}$. La littérature est exploration du bruit, dont le sens est au $\mathrm{xx}^{\mathrm{e}}$ siècle encore trop encrypté.

Pour Kittler, peu importent les écoles, dadaïstes, expressionnistes, surréalistes. Il cite Morgenstern, Kafka, Heym, Ball, van Hoddis, Huelsenbeck, Urzidil, Rilke, Adler, Breton, et le jeune G. Benn, qui développant une comptabilité à double entrée, se met à écrire avec des statistiques dans une main tout en délirant avec l'autre. Alors que le mot a cessé d'avoir une fonction poétique, pour reprendre l'expression de Hugo Ball, le Nombre constitue une bouée de secours. Le bruit est encodé et le poète a pour tâche de le décoder, sinon de le déchiffrer. Au xxe siècle, les dés ne cesseront pas d'être lancés. La lettre redevient - comme c'était le cas chez Pythagore - ce maitre de musique et de poésie $^{41}$, un nombre combinable, permutable et une inconnue. Dès 1905 Morgenstern compose Fisches Nachtgesang ( $L a$ prière nocturne du poisson), un poème uniquement constitué de signes métriques, l'agencement des longues et des brèves permettant de faire apparaître un poisson sur la page. En 1913, Vélimir Khlebnikov et Alexeï Kroutchenykh écrivent La lettre en tant que telle, et en 1914, Marinetti publie à Rome $L a$ Splendeur géométrique et mécanique et la sensibilité numérique. Au même moment, Marcel Duchamp transforme avec ses ready-made l'art en jeu d'échecs. L'un des maîtres de Dada, auquel Kittler ne consacre jamais plus que quelques mots - peut-être parce qu'il 
est déjà du côté du cinéma et non plus du gramophone -, Hugo Ball assène en 1916 que le vers est sans mots. Le poème moderne, fait de bruits et de nombres, s'écrit automatiquement par l'inconscient, claque comme une machine à écrire et se lit avec le corps tout entier. Artistes visuels, sonores, performeurs - l'art de la performance naît précisément ces années-là, chanteurs et poètes - tombés de l'olympe - appartiennent désormais au même monde. Celui des machines. L'auteur 1900 est un artiste comme les autres $^{42}$, l'artiste peut s'emparer pleinement du langage. Tandis que les morceaux des groupes pop (les Beatles, les Rolling Stones, les Pink Floyd) célèbrent l'avènement des machines et sont élevés - chez Kittler - au rang de textes littéraires - ce que le jury du Nobel finira par reconnaître, il reste au romancier d'expérimenter l'écriture automatique par couper-coller, préfigurant la fonction copier-coller de nos ordinateurs (William S. Burroughs), ou de fouiller leur(s) histoire(s) souterraines (Thomas Pynchon).

Les média techniques chantent. Les hackers le savent. L'un des premiers d'entre eux, le phreaker John Draper, alias Captain Crunch, piratait le réseau du téléphone en produisant une fréquence de $2600 \mathrm{~Hz}$ à partir d'un sifflet trouvé dans une boîte de céréales. Dans les années 1980, les hackers du réseau Minitel simulaient des microserveurs en utilisant des cartes sons. En transmission serveur-terminal, le bit « 1 » correspond à une fréquence de $1300 \mathrm{~Hz}$ et le bit « 0 » à $2100 \mathrm{~Hz}$. En voie retour, « 1 » correspond à $390 \mathrm{~Hz}$ et « 0 » à $450 \mathrm{~Hz}$. Le lien intime entre la musique - le chant des sirènes - et les nombres a occupé les dernières années de Kittler ${ }^{43}$. Il y défend l'idée que tout ce qui définit la culture occidentale est d'origine érotique - amour et sexe : les mots, l'éducation, la science, les dieux - les média. " Aphrodite, bleib uns nah ${ }^{44}$ » conclut le premier volume. Conçu comme une tétralogie dont deux volumes seulement ont paru, l'ouvrage prétend comprendre l'origine de l'art et de la connaissance par l'analyse de la culture grecque tout entière dont Pythagore, le quintuple inventeur de l'art de compter, de chanter, d'enseigner, de vivre et de mourir, constitue la figure emblématique - avec Ulysse. Dans ce monde où les déesses, où Circé, Calypso, et l'alphabet devenu nombres chantent, où au son des quatre îles et des cinq Sirènes du désir, les Grecs ont tissé - le tissage, cette activité féminine - c'est-à-dire encodé et chiffré - le réel de l'Occident. Les média et les réseaux de communication numériques, par l'éternel retour de l'histoire, nous renvoient à cette histoire lointaine du premier coup de dés. Quand l'auteur 1900 faisait «parler » l'inconscient, celui du système 2000, s'il existe, n'a d'autres choix que de « faire parler » et « chanter » les machines.

En 1965, alors que les mouvements de l'art optique et de l'art cinétique sont à la mode à Paris et dans le monde de l'art en général, Georg Nees utilise une machine à dessiner Graphomat Z64, que Siemens avait achetée l'année précédente à Zuse KG pour réaliser dans la plus grande confidentialité une série d'impressions qu'il expose à la Studien Galerie de l'Université Technique de Stuttgart. Il s'agit de la première exposition d'art numérique connue. Georg Nees est un étudiant du professeur de philosophie Max Bense, auteur en 1949 de Die Mathematik in der Kunst. Quelques années auparavant, en 1959, un autre étudiant de M. Bense, un jeune informaticien nommé Theo Lutz, dans le même lieu et dans la même université crée, avec un Zuse Z 22, une base de données contenant 16 sujets et 16 prédicats extraits du Château de Kafka et programme la première œuvre littéraire ${ }^{45}$ produite avec un ordinateur numérique : Stochastische Texte. Un générateur de texte qu'il publie la même année dans Augenblick ${ }^{46}$, la revue littéraire et philosophique fondée par Bense en 1955. Si « la mort de l'auteur » du système 1800 semble être une préoccupation française, l'émergence de l'auteur du système 2000 est 
une affaire allemande. Alors que le style en art devient affaire de code, de commutation et de flux, l'«auteur» de notre époque n'est plus que le co-auteur de ses œuvres, cherchant avant tout à comprendre ce nouvel Autre qui fait office à la fois de secrétaire et de lecteur, et contraint de renoncer à ce statut de créateur qu'il avait cru obtenir au XIX ${ }^{e}$ siècle avec la mort de Dieu.

\section{Un téléphone qui parlerait à un gramophone (Kafka) et la disparition de la communauté de lettrés}

20 Mais la poésie numérique ou sonore, pas plus que les calligrammes de Guillaume Apollinaire ou les poèmes de Christian Morgenstern ne se lisent comme on lisait au début $d u \mathrm{XIX}^{\mathrm{e}}$ siècle, c'est-à-dire ne se traduisent en signifiés. Cette manière d'écrire nécessite une autre méthode de lecture que l'interprétation. «Un médium est un médium. Il ne peut donc pas être traduit. Transmettre des messages d'un médium à un autre médium signifie toujours: le soumettre à d'autres standards et d'autres matérialités ${ }^{47}$ ». En même temps que l'auteur 1800, disparaît son lecteur ou plutôt sa lectrice, ce sujet-interprète capable d'accéder à un monde de bruits et d'images pardelà les lettres. À sa place, un appareil récepteur, transposant des matérialités dans d'autres matérialités, des ondes sonores en ondes électriques ou encore des signifiants en d'autres signifiants. Pratiquement, de quoi s'agit-il ? Friedrich Kittler cite une lettre de Kafka à sa fiancée Félice Bauer (à qui il écrivait des lettres typographiées), où prenant acte du court-circuit média-technique, il imagine «une connexion directe entre un parlographe, "qui irait au téléphone à Berlin » pour " un petit entretien », et un "gramophone à Prague ${ }^{48}$ ", laissant entendre que dans cette disparition du lecteur 1800, c'est le livre même qui serait menacé. L'échange de lettres entre amis qui ne se connaissent pas, autrement dit, la littérature universelle des classiques, n'est plus le fondement ni de l'éducation ni des sociétés, écrit radicalement Peter Sloterdijk ${ }^{49}$. Le système 1900 est l'instauration d'un nouveau rapport au canon littéraire et de nouveaux préceptes éducatifs: en Allemagne, le réformisme pédagogique [Reformpädagogik] soutenu par l'empereur Guillaume II voit se développer le mouvement de l'éducation par l'art [Kunsterziehungsbewegung] selon lequel l'enseignement fondé sur les faits et les expériences doit prendre le pas sur l'accumulation de connaissances mémorisées, ou encore, en cours d'allemand, les essais libres écrits par les élèves doivent remplacer le commentaire des œuvres classiques ${ }^{50}$. L'auteur et son œuvre ne sont plus le fondement de l'éducation. Le commentaire répétition masquée conférant au texte le statut de discours réactualisable à l'infini n'est plus constitutif du nouvel ordre du discours ${ }^{51}:$ l'élève ne doit pas lire, il doit écrire sans peur de ne pas égaler les modèles anciens, sans entrave. Écriture automatique, traduit Kittler, écriture de l'inconscient : « les essais libres de la classe d'allemand sont un exercice de couplage de deux phrases impossibles j'écris et je délire ${ }^{52} »$.

21 La lecture elle-même en effet n'est pas le processus immatériel qu'elle prétendait être dans le système 1800. Alors que le réformisme éducatif en appelle à une prise en compte psycho-physique de l'enfant, la lecture est désormais objet d'étude expérimental. Le processus de lecture acquiert une positivité psycho-physique tout à fait nouvelle, qui en fait un objet d'étude indépendant de toute pensée ou compréhension $^{53}$. Ce qu'il y a par exemple de remarquable dans la St-Georg Schrift est qu'elle prenne en compte les critères psycho-physiques de la lisibilité et de la 
reconnaissance des lettres, tels qu'ils sont dégagés au début $d u x^{e}$ siècle par plusieurs expérimentations et mesures, en particulier celles réalisées au moyen du tachistoscope, permettant de mesurer en temps et en intensité la présentation visuelle des stimuli. Les psychologues-ingénieurs Benno Erdmann et Raymond Dodge ${ }^{54}$ établissent ainsi qu'une lecture élémentaire ne perçoit pas les lettres de l'alphabet, mais seulement les différences entre elles, et que la reconnaissance des mots s'appuie sur des lettres isolées et discontinues qui font saillie. Les mots sont reconnus par le système nerveux grâce à leur forme d'ensemble, c'est-à-dire aux traits "par lesquels le dessin noir des lettres contraste avec l'espace blanc de l'arrière-fond ${ }^{55}$ » par le système nerveux. Les consonnes qui "dépassent" sur les lignes du haut ou du bas sont des signaux typographiques de reconnaissance et non des signes renvoyant à un sens, les lettres de l'alphabet sont perçues comme des éléments discrets et non dans l'unité du mot qu'elles forment. Le but recherché par Stefan George n'est cependant pas l'aisance de la lecture mais la résistance de la lettre écrite au regard, l'irréductibilité de la matérialité à toute représentation qui s'en éloignerait et prétendrait la congédier.

Si la lecture persiste, elle ne peut plus être une herméneutique. Les matérialités de l'écrit exigent « une interprétation objective dont le seul modèle sont les techniques de décodage en cryptographie ${ }^{56} »$. La méthode freudienne d'interprétation des rêves, par exemple, n'est pas une interprétation, mais une «transposition de médium qui transforme les images et les bruits en lettres ${ }^{57} »-$ puis les lettres en livres. Tout l'art du psychanalyste consiste précisément à ne pas donner sens aux paroles du patient, à refréner «toutes les objections logiques et affectives qui veulent l'inciter à faire une sélection $^{58}$ " afin de ne pas projeter sur les paroles délirantes une censure personnelle qui en évacuerait le non-sens en même temps qu'elle empêcherait toute écoute de l'inconscient. Tel est le sens de l'injonction freudienne à devenir récepteur de téléphone $e^{59}$, qui enregistre, transforme les ondes sonores en oscillations électriques, mais n'interprète pas les voix au bout du fil. Si Freud fait une sélection, c'est une sélection de signifiants qu'il interprète comme un rébus. Interpréter un rêve, comme l'explique Freud au début de L'Interprétation du rêve, ce n'est pas lui substituer un sens global, tout fait. Ce n'est pas non plus le décomposer en des éléments distincts qui auraient chacun un sens, c'est dégager le système de relations qui structure les signifiants ${ }^{60}$ et qui est chaque fois particulier à un discours et à un patient. Le rêve est un rébus : l'image est à prendre comme un signe, qui peut être aussi bien un mot, qu'une syllabe, une lettre ou une marque de ponctuation. Les signifiants ne font signe vers aucun signifié universel : ils ne valent que dans un système de renvois internes au discours du patient que le psychanalyste ne peut décrypter qu'au cas par cas. Le discours du patient est un système différentiel d'éléments matériels discrets.

La méthode interprétative n'est qu'une des modalités possibles de la transposition de médium. Ce que Friedrich Kittler reproche aux études de germanistique est de n'avoir pas pris acte de la fin du système auteur-lecteur de 1800 et de lire la littérature postérieure comme si le système d'inscription et les conditions média-techniques de sa production étaient restés inchangés. Ainsi les chercheurs en littérature ont-ils identifié dans la sérialité des Cahiers de Malte Laurids Brigge de Rilke des «symétries artistiques, des ordres, des lois de compositions " intentionnelles, là où il n'y avait qu'écriture automatique, Brigge notant « les phénomènes les plus simples d'après d'élémentaires règles aléatoires ${ }^{61}$ ». Ce que la littérature désormais enregistre, et ce que le lecteur doit décoder, est « le déchet » $<A b f a l l>$ que laissent les corps et l'inconscient sur le papier à travers les processus de traitement de l'information à l'œuvre, processus matériels 
d'inscription que la lecture savante critiquée par Kittler ignore, en même temps qu'elle ignore son propre outillage. La critique est radicale, car la lecture intensive mobilise immanquablement les règles grammaticales, orthographiques, ou encore le contexte historique et culturel pour faire surgir le sens d'un texte, et en ce sens explore l'inconscient du texte. Mais le cœur du propos est ailleurs: le texte se construit précisément dans et avec ce qui ne fait pas sens, et la réception du livre ne peut se réduire à l'ordre du symbolique. L'horizon de la lecture savante intensive, ce sens qu'il s'agirait d'expliciter et qui serait intuitivement accessible sans médiation, vole en éclat: si de toute manière les matérialités du livre ne sont jamais transposables sans être trahies, il ne peut plus y avoir que des transpositions plurielles, aussi incomplètes les unes que les autres. C'est donc en même temps le monopole de certains types de lectures savantes et non savantes, qui est remis en question. Avec la généralisation des supports numériques d'écriture et de lecture (hyper-lecture, lecture assistée par ordinateur $\left.{ }^{62}\right)$, le débat ouvert en 1900 sur les pratiques universitaires et pédagogiques n'a pas fini de se poursuivre.

\section{BIBLIOGRAPHIE}

Balpe Jean-Pierre, http://www.balpe.name/+-Generateur-de-texte-en-ligne-

Barthes Roland, « La mort de l'auteur », in Le Bruissement de la langue, Paris, Le Seuil, 1984.

Bense Max, Die Mathematik in der Kunst, Hambourg, Claassen \& Goverts, 1949.

Birot Pierre-Albert, Poème à crier et à danser [1917], in SIC (Sons Idées Couleurs, Formes), Pierre Albert-Birot (dir.), Paris, Sic, 1919.

Bootz Philippe, Les Basiques, La littérature numérique : https://www.olats.org/livresetudes/ basiques/litteraturenumerique/basiquesLN.php

Braffort Paul, http://www.paulbraffort.net/litterature/alamo/alamo_experience.html

Chklovski Victor, L'Art comme procédé [1917], trad. R. Gayraud, Paris, Allia, 2008.

Derrida Jacques, De la grammatologie, Paris, Les Éditions de Minuit, 1967.

Edelstein-Gomez Madja, https://lesrecombinants.fr

Foucault Michel, « Qu'est-ce qu'un auteur ?» [1969], Dits et écrits, vol. I., Paris, Gallimard, 2001.

_-_, L'Ordre du discours, Paris, Gallimard, 1971.

-- , Les Mots et les choses [1966], Paris, Gallimard, 2003.

Freud Sigmund, L'Interprétation du rêve [1900], trad. J. Altounian, P. Cotet, et al., Paris, PUF, 2013.

Guess Jeff, https://twitter.com/FKittlerbot

Hayles Katherine, How We Became Posthuman, Chicago, The University of Chicago Press, 1999.

Kittler Friedrich, «Le logiciel n'existe pas » [1993] in Mode protégé, trad. F. Vargoz, Dijon, Presses du réel, 2015. 
-_-, Aufschreibesysteme 1800-1900 [1985], 3e édition, Munich, Wilhelm Fink Verlag, 1995.

-_-, Gramophone, Film, Typewriter [1986], Dijon, à paraître aux Presses du réel, dernier semestre 2017.

-_-, Médias optiques. Cours berlinois 1999, trad. dirigée par Audrey Rieber (dir.), Paris, L'Harmattan, coll. « Esthétiques allemandes », 2015.

-_-, Musik und Mathematik, Munich, W. Fink Verlag, vol. 1, parties I et II, 2006-2009.

---, Unsterbliche. Nachrufe, Errinnerungen, Geistergespräche, Munich, W. Fink Verlag, 2004.

Lacan Jacques, Écrits, Paris, Le Seuil, 1966.

-—-, Les Écrits techniques de Freud, Paris, Le Seuil, 1998.

Lutz Theo, «Stochastische Texte », in Augenblick, 4, 1959 : https://auer.netzliteratur.net/0_lutz/ lutz_original.html

Maigret Nicolas, https://twitter.com/predartbot

Mallarmé Stéphane, La Littérature. Doctrine, in CEuvres complètes, H. Mondor et G. Aubry (dir.), Paris, Gallimard, coll. « Bibliothèque de la Pléiade », 1945.

Meillassoux Quentin, Le Nombre et la sirène, Paris, Fayard, 2011.

Nietzsche Friedrich, « Lettre de fin février 1882 », in Briefwechsel, III-1, G. Colli et M. Montinari (eds), Berlin, Walter de Gruyter, 1975-1984.

Schlegel August Wilhelm, Vorlesungen über schöne Literatur und Kunst [1801], Éditions Jacob Minor, Heilbronn, Henninger, 1884.

Sloterdijk Peter, Règles pour le parc humain [1999], trad. O. Mannoni, Paris, Fayard, 2000.

Tullett Barrie, Typewriter Art, A Modern Anthology, Londres, Laurence King Publishing, 2014.

Wardrip-Fruin Noah, « Digital Media Archaeologies: Interpreting Computational Processes », in Erkki Huhtamo et Jussi Parikka (eds), Media Archaeology: Approaches, Applications, and Implications, Berkeley, University of California Press, 2011.

\section{NOTES}

1. Kittler Friedrich, « Le logiciel n'existe pas » [1993], in Mode protégé, trad. F. Vargoz, Dijon, Presses du réel, 2015, p. 30.

2. Foucault Michel, « Qu'est-ce qu'un auteur?» [1969], Dits et écrits, vol. I., Paris, Gallimard, 2001, p. 789-821.

3. Kittler F., Gramophone, Film, Typewriter [1986], Dijon, Presses du Réel, à paraître au dernier semestre 2017, p. 124 sq.

4. Ce terme traduit le concept central dans la pensée de Friedrich Kittler d'" Aufschreibesystem ». Dans la postface à la seconde édition de son ouvrage Aufschreibesysteme 1800-1900, il explique comment ce terme, qu'il emprunte aux Mémoires d'un névropathe de Daniel Paul Schreber et qui signifie littéralement « système de prises de notes ", peut aussi désigner un « réseau technique et institutionnel permettant à une culture donnée d'adresser, de stocker, de traiter des données pertinentes ». C'est pour rendre ce sens que nous avons choisi le terme inscription, qui peut en français, faire référence à tous les types d'enregistrement. 
5. Kittler F., Aufschreibesysteme 1800-1900 [1985], 3e édition, Munich, Wilhelm Fink Verlag, 1995, p. 140. (trad. F.V.) C'est nous qui traduisons les citations des œuvres de Kittler non traduites en français.

6. Kittler F., Gramophone, Film Typewriter, op. cit., p. 129.

7. Ibid., p. 103.

8. Derrida Jacques, De la grammatologie, Paris, Les Éditions de Minuit, Paris, 1967, p. 33 et sq.

9. Schlegel August Wilhelm, Vorlesungen über schöne Literatur und Kunst [1801], Jacob Minor (ed.), Heilbronn, Henninger, 1884, en réponse à la question « Qu'est-ce-que la poésie? ", cité par F. Kittler in Aufschreibesysteme 1800-1900, p. 204.

10. Foucault Michel, Les Mots et les choses [1966], Paris, Gallimard, 2003, p. 298, cité par

F. Kittler in Aufschreibesysteme 1800-1900, op. cit., p. 43.

11. Kittler Friedrich, Aufschreibesysteme 1800-1900, op. cit., p. 44. (trad. F.V.)

12. Kittler F., Gramophone, Film, Typewriter, op. cit., p. 158.

13. Ibid., p. 171.

14. Kittler F., Unsterbliche. Nachrufe, Errinnerungen, Geistergespräche, Munich, Wilhelm Fink Verlag, 2004, p. 123. (trad. E.G.)

15. Cf. Lacan Jacques, Les Écrits techniques de Freud, Paris, Le Seuil, 1998, p. 125-129.

16. Lacan Jacques, Écrits, Paris, Le Seuil, 1966, p. 109.

17. Cf. Kittler Friedrich, Médias optiques. Cours berlinois 1999, trad. Anaïs Carvalho, Tamara Eble, Ève Vayssière et Slaven Waelti, traduction dir. par Audrey Rieber, Paris, L'Harmattan, coll. « Esthétiques allemandes », 2015, p. 61 : «On refusera systématiquement toute tentative de définir une fois de plus l'âme ou l'homme [...] la seule chose que l'on peut en connaître ce sont les appareils techniques par lesquels l'âme ou l'homme prennent à chaque époque la mesure d'eux-mêmes. »

18. Nietzsche Friedrich, "Lettre de fin février 1882 », in Briefwechsel, III-1, G. Colli et M. Montinari (eds), Berlin, Walter de Gruyter, 1975-1984, p. 172. (trad. F.V.)

19. «Le signifié minimal murmuré par la parole source ne se préserve qu'à condition de ne pas être dit, d'où la nécessité de la mine de plomb », F. Kittler, Aufschreibesysteme 1800-1900, op. cit., p. 81. (trad. F.V.)

20. La première machine utilisée par Nietzsche, après que l'aggravation de sa cécité l'eut empêché d'écrire à la main, fut la « sphère écrivante » de Mailing Hansen, qui ne permet pas au dactylographe de lire ce qu'il écrit ». Cf. Gramophone, Film, Typewriter, op. cit., p. 346-349.

21. Cf. Barthes Roland, « La mort de l'auteur », in Le Bruissement de la langue, Paris, Le Seuil, 1984, p. 64-65. Et notamment cette phrase : «En France, Mallarmé sans doute le premier, a vu et prévu dans toute son ampleur la nécessité de substituer le langage luimême à celui qui jusque-là en était le propriétaire ; pour lui comme pour nous, c'est le langage qui parle [...] Toute la poétique de Mallarmé consiste à supprimer l'auteur au profit de l'écriture. »

22. Ibid., p. 66.

23. Mallarmé Stéphane, La Littérature. Doctrine, in Euvres complètes, H. Mondor et G. Aubry (dir.), Paris, Gallimard, coll « Bibliothèque de la Pléiade », 1945, p. 850, cité par Kittler in Gramophone, Film, Typewriter, op. cit., p. 47.

24. Kittler F., Aufschreibesysteme 1800-1900, op. cit., p. 256.

25. Cf. l'analyse de Quentin Meillassoux soutenant l'hypothèse que le Nombre (crypté) du Coup de dés est 707. Meillassoux Quentin, Le Nombre et la Sirène, Paris, Fayard, 2011.

26. Kittler F., Gramophone, Film, Typewriter, op. cit., p. 61. 
27. Cf. Kittler F., Aufschreibesysteme 1800-1900, op. cit., p. 314 et sq.

28. Kittler F., Gramophone, Film, Typewriter, op. cit., p. 380.

29. Par le formalisme russe, par exemple Viktor Chklovski montrant dans L'Art comme procédé que l'objet d'art se construit du côté de la réception, puis par l'école de Prague (Jan Mukarovsky).

30. Kittler F., Aufschreibesysteme 1800-1900, op. cit., p. 229. (trad. F.V.)

31. Ibid., p. 230.

32. Ibid., p. 261.

33. Ibid., p. 264.

34. L'ALAMO (Atelier de Littérature Assistée par la Mathématique et les Ordinateurs) a été fondé en 1982 par Paul Braffort et Jacques Roubaud. Cf. http://

www.paulbraffort.net/litterature/alamo/alamo_experience.html (consulté le 1er septembre 2017).

35. Cf. Hayles Katherine, How We Became Posthuman, Chicago, The University of Chicago Press, 1999.

36. Kittler F., Aufschreibesysteme 1800-1900, op. cit., p. 385.

37. Ibid., p. 423.

38. Cf. les générateurs de textes littéraires de Jean-Pierre Balpe. Par exemple, http:// www.balpe.name/+-Generateur-de-texte-en-ligne- (consulté le 1er septembre 2017). Pour une synthèse de la littérature numérique, voir Philippe Bootz, Les Basiques, La littérature numérique : https://www.olats.org/livresetudes/basiques/

litteraturenumerique/basiquesLN.php (consulté le 1er septembre 2017).

39. Cf. par exemple, le FKittlerbot de Jeff Guess, générant du texte à partir de Gramophone, Film, Typewriter (éd. anglaise) (https://twitter.com/FKittlerbot), le Predictive Art Bot de Nicolas Maigret (https://twitter.com/predartbot) ou Les Recombinants de Madja Edelstein-Gomez (https://lesrecombinants.fr) (tous consultés le 1er septembre 2017).

40. Cf. Birot Pierre-Albert, Poème à crier et à danser (1917). Nous remercions ici Isabelle Kryzwkowski pour nous avoir fait connaître ce poème et Dorothée (Doe) Polanz pour l'avoir lu. Cf. Isabelle Kryzwkowski, « Esthétique de la surprise et conscience du medium : la construction de l' « émersion », des avant-gardes historiques aux littératures numériques », conférence du 5 juin 2016, in Colloque « Archéologie des media et écologies de l'attention », Cerisy-la-Salle, mai-juin 2016.

41. Cf. Kittler Friedrich, Musik und Mathematik, vol. 1, Munich, W. Fink Verlag, 2006. 42. Cf. dès les années 1900 les dessins de Flora F. F. Stacey et G. M. Patterson. Le procédé qui consiste à penser, avec la machine à écrire, les mots comme des images et les lettres comme des lignes est exploré par le mouvement De Stijl dans les années 1920 et constitue une technique majeure de la poésie concrète. Voir à ce sujet Barrie Tullett, Typewriter Art, A Modern Anthology, Londres, Laurence King Publishing, 2014.

43. Cf. Kittler F., Musik und Mathematik, Munich, W. Fink Verlag, 2006 pour le vol. 1 « Hellas », $1^{\text {re }}$ partie; « Aphrodite »; 2009 pour le vol. 1, 2 e partie : « Eros ».

44. Ibid., vol. 1, p. 337.

45. Il est vrai que, comme le rappelle Noah Wardrip-Fruin, la première œuvre de littérature numérique pourrait être les Lettres d'amour, nées de la rencontre entre Alan Turing et Christopher Strachey. Programmées en 1952 sur l'ordinateur chéri par Turing, le Mark I de Manchester, plus connu sous le nom de M.U.C., les Lettres d'amour paraissent en 1954 dans la revue conservatrice Encounter (« The Thinking Machine », Encounter, October 1954, p. 25-31). Cf. Noah Wardrip-Fruin, « Digital Media 
Archaeologies: Interpreting Computational Processes ", in Erkki Huhtamo et Jussi Parikka (eds), Media Archaeology: Approaches, Applications, and Implications, Berkeley, University of California Press, 2011. Enfin, il faut noter qu'en 1954 Brion Gysin compose sur un ordinateur Honeywell son poème sonore I am that I am. Entre l'Allemagne, les États-Unis et le Royaume-Uni, la querelle de l'invention de l'ordinateur se poursuit jusque dans l'histoire de la littérature.

46. Lutz Theo, «Stochastische Texte », in Augenblick, 4, 1959 : https:// auer.netzliteratur.net/0_lutz/lutz_original.html (consulté le 1er septembre 2017). 47. Kittler F., Aufschreibesysteme, op. cit., p. 335. (trad. F.V.)

48. Kittler F., Gramophone, Film, Typewriter, op. cit., p. 377.

49. Cf. Sloterdijk Peter, Règles pour le parc humain [1999], trad. O. Mannoni, Paris, Fayard, 2000, p. 12-13. «[...] L'art d'écrire des lettres inspirant l'amour à une nation d'amis, quel que soit le professionnalisme avec lequel on l'exerce, ne suffirait plus à établir le lien télécommunicatif entre les habitants d'une société de masse moderne. Avec l'établissement médiatique de la culture de masse dans le monde industrialisé après 1918 (radio) et après 1945 (télévision), et plus encore avec les révolutions actuelles des réseaux, on a donné de nouvelles bases à la coexistence des êtres humains dans les sociétés actuelles. »

50. Sur tous ces points, cf. Kittler F., Aufschreibesysteme 1800-1900, op. cit., p. 389-393 et p. $398-400$.

51. Cf. Foucault Michel, L'Ordre du discours, Paris, Gallimard, 1971, p. 27-28.

52. Quand, selon Michel Foucault, l'époque classique (qui embrasse le système d'inscription 1800) affirmait bien au contraire : "Là où il y a œuvre, il n'y a pas folie ", cf. Michel Foucault, Histoire de la folie à l'âge classique, Paris, Gallimard, 1972, p. 557.

53. Cf. Moisseiff Leon S. et Gertrude Stein, « Normal motor Automatism », in Psychological Review, 3, 1896, p. 492-512, cité par F. Kittler in Aufschreibesysteme 1800-1900, op. cit., p. 258 : «We may sum up the experiment by saying that a large number of acts ordinarily called intelligent, such as reading, writing, etc., can go quite automatically in ordinary people.»

54. Cf. Benno Erdmann et Raymond Dodge, Psychologische Untersuchungen über das Lesen auf experimenteller Grundlage, Halle, Niemeyer, 1898, cité par Friedrich Kittler in Aufschreibesysteme 1800-1900, op. cit., p. 319.

55. Kittler F., Aufschreibesysteme 1800-1900, op. cit., p. 319. (trad. F.V.)

56. Ibid., p. 340.

57. Ibid., p. 359.

58. Freud Sigmund, « Conseils au médecin dans le traitement psychanalytique » [1912], trad. R. Lainé, in La Technique psychanalytique, Paris, PUF, 2007, p. 75.

59. Loc. cit.

60. Cf. Freud Sigmund, L'Interprétation du rêve [1900], trad. J. Altounian, P. Cotet, et al., Paris, PUF, 2013, p. 131-132.

61. Kittler F., Aufschreibesysteme 1800-1900, op. cit., p. 402. (trad. F.V.)

62. Sur les différents types de lecture et la manière dont les média numériques affectent les pratiques de lecture, cf. Hayles Katherine, Lire et penser en milieux numériques. Attention, récits, technogenèse, trad. C. Degoutin, Grenoble, ELLUG, Grenoble, 2016, p. 119-156. 


\section{RÉSUMÉS}

En 1900, Stéphane Mallarmé, Christian Morgenstern, Hugo Ball ou encore Stefan George bouleversent la création littéraire en produisant des œuvres qui semblent s'affranchir du sens en même temps qu'elles expérimentent les matérialités de la page, des lettres ou des sons. Une partie de la littérature ne se préoccupe plus d'ouvrir à un monde de l'imagination accessible pardelà les lettres écrites, et explore au contraire l'écriture automatique ou le pouvoir de signifiants qui font obstacle à l'intentionnalité de l'acte d'écriture. Mais un auteur qui ne se reconnaît plus dans ce qu'il écrit est-il encore un auteur ? Si à l'âge classique folie et littérature s'excluaient, le $\mathrm{xx}^{\mathrm{e}}$ siècle est le siècle d'une écriture qui s'écrit toute seule, et qui à ce titre échappe à l'exigence du sens pour devenir un simple jeu de symboles, c'est-à-dire un code qui ne peut se déchiffrer qu' a posteriori. Pour Friedrich Kittler, l'auteur est une fonction de son environnement médiatechnique et celui de 1900 est façonné par l'apparition du phonographe, qui enregistre ce que l'écriture échouait à dire (les bruits), du cinéma qui convoque l'imaginaire en agissant directement sur le système nerveux et de la machine à écrire qui permet d'écrire plus vite que l'on ne pense. Si tout s'écrit, que faut-il lire et comment? Le tournant du XIXe siècle est aussi l'époque en Europe d'une réforme pédagogique qui encourage les libres essais des élèves et non plus la lecture des auteurs classiques. La "mort de l'auteur ", énoncée par Roland Barthes et Michel Foucault, est ainsi en réalité pour Friedrich Kittler celle de l'auteur de ce qu'il appelle le système d'inscription 1800 , c'est-à-dire le réseau institutionnel et technique déterminant les modalités selon lesquelles les données peuvent être, à cette époque, adressées, stockées et traitées. Ce qui disparaît est le monopole d'une conception herméneutique de la lecture qui faisait d'un monde d'idées universelles le fondement et l'horizon de la littérature et de la lecture, pour laisser place aux matérialités de l'écriture et à une prise en compte renouvelée de la visualité de la page graphique.

\section{INDEX}

Mots-clés : auteur, mort de l'auteur, système d'inscription, système d'enregistrement, écriture, lecture, sujet, imaginaire, théorie des média, littérature numérique

\section{AUTEURS}

\section{EMMANUEL GUEZ}

Emmanuel Guez est artiste, théoricien des média et codirecteur du laboratoire de recherche PAMAL (Preservation \& Art - Media Archaeology Lab) à l'École supérieure d'art d'Avignon.

\section{FRÉDÉRIQUE VARGOZ}

Frédérique Vargoz est Professeur de philosophie au lycée français de Vienne. Frédérique Vargoz est rattachée à l'université Grenoble Alpes, PPL, F-38000 Grenoble 